\title{
Design and Implementation of a Highly Efficient VLSI Architecture for Discrete Wavelet Transform'
}

\author{
Chu Yu, Chien-An Hsieh, and Sao-Jie Chen \\ Department of Electrical Engineering \\ National Taiwan University, \\ Taipei, Taiwan, R.O.C.
}

\begin{abstract}
Since the discrete wavelet transform (DWT) is a kind of multi-rate transform, it is difficult to design an optimal computation-time architecture for the DWT. In this paper, we propose a highly efficient VLSI architecture for the 1-D DWT decomposition. This architecture contains two stages of systolic decimation filter banks to guarantee a high throughput and an optimal computation time. Using this architecture, $N$-point samples with $J$ resolution levels can be computed in $N$ clock cycles spending only $J L$ registers, where $L$ denotes filter length. Due to its regular structure, this architecture can be easily scaled up with the tap size of the filters and the number of octaves. The performance of the proposed architecture will be verified by the successful implementation of a 4-tap 3-octave DWT VLSI chip.
\end{abstract}

\section{Introduction}

The discrete wavelet transform provides a new method for signal processing [1]-[2]. It decomposes data into components of different frequencies, such that we can have good time resolution at high frequencies and good frequency resolution at low frequencies. The wavelet transforms are well suited for analyzing physical situation where signal contains discontinuities and sharp spikes. Recent developments have led the DWT into many applications such as audio and image compression, image recognition system, transient signal analysis, computer graphics, and so on.

For real-time and high-speed applications, a dedicated DWT hardware device is needed and several VLSI architectures have been proposed [3-6]. Knowles [3] proposed the first VLSI architecture for the 1-D DWT. Later, Parhi and Nishitani [4] proposed a folded and a digit-serial architectures for the 1-D DWT. The latter two architectures have fixed-size DWT octave, and are not designed to scale up with the number of octaves and the size of the filters. Therefore, Fridman and Manolakos[5] proposed a multiprojection based systolic architecture for the 1-D DWT. In addition, Vishwanath ot al. [6] proposed three routing-network based systolic architectures for the 1-D DWT. These

${ }^{\top}$ This work was supported by National Science Council, unde grants NSC 86-221 5-E002-034 and NSC 86-2221-E002-066
1-D DWT architectures [5]-[6] are scalable, but their input sequence has to interleave, thus deriving lower throughput.

In this paper, we present a scalable VLSI architecture which can compute data on-the-fly, i.e., the input data can be processed at the rate of one sample per clock cycle. In our design, all the computations of DWT. except those of the first octave, are folded into the computations of the second octave. Thus, this folded architecture can provide an ideal high throughput and still retain its scalability. In addition, a 4-tap 3-octave DWT chip has been implemented to verify the performance of the proposed architecture.

\section{Discrete Wavelet Transform}

The wavelet transform (WT) is a kind of time-scale decomposition of signals. The WT and Short-time Fourier transform (STFT) differ in their time-frequency representations. The WT processes data with different window widths at different scales (frequencies), which overcomes the limitation of fixed time-frequency resolution of STFT.

The discrete wavelet transform of a signal $x(t)$ is given by:

$$
W(b, a)=\frac{1}{\sqrt{|a|}} \sum x(t) \cdot h\left(\frac{1-b}{a}\right)
$$

where $b$ is the time factor, $a$ is the scale factor, $h(t)$ is the wavelet basis function. Properties of wavelet transforms are heavily dependent on their basis wavelet functions.

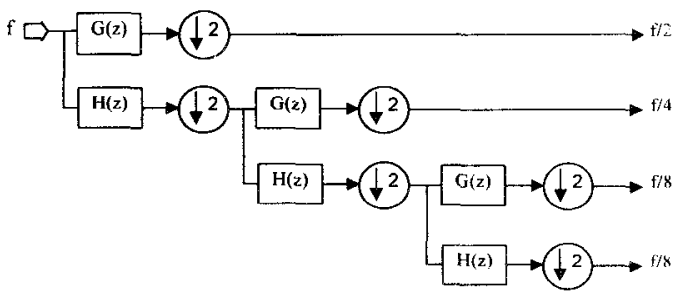

Fig. 1 A 3-octave filter bank tree for the 1-D DWT

The DWT can also be viewed as a kind of multiresolution decomposition of a sequence. By exploring the subband scheme recursively, a fast DWT can be constructed. Figure 1 shows a three-octave filter bank tree for the 1-D DWT, where $H(z)$ and $G(z)$ represent a low-pass and a high-pass filters, respectively; and $\downarrow 2$ represents subsampled by 2 , by 
dropping one every two samples. Assume an input sequence $x(n)$ contains $N$ samples, then the output sequence length should also be $N$. The first octave computes $N / 2$ samples, the second octave computes $N / 4$ samples, $\cdots$, and so on.

\section{VLSI Architecture for DWT}

As mentioned above, a scalable architecture is our design goal. Therefore, in this section we present a novel and efficient VLSI architecture for the 1-D DWT decomposition, which performance is comparable with other previously proposed scalable architectures [5]-[6]. The overall architecture is given in the following subsection and the decimation filter scheme is described in Subsection 3.2.

\subsection{Overall Architecture}

As shown in Fig. 1, we need to compute $N$-point samples in the first octave, then generate $N / 2$ output samples. Similarly, in the second octave, we need to compute $N / 2$ input samples and generate $N / 4$ output samples. For an $m$ octave DWT, the total number of samples to compute is:

$$
N+\left(\frac{1}{2}\right) N+\left(\frac{1}{2}\right)^{2} N+\cdots+\left(\frac{1}{2}\right)^{m-1} N=2 N\left(1-2^{-m}\right)<2 N
$$

Assume one filter bank can compute $N$ samples in one unit time, at most two filter banks are needed to generate all the discrete wavelet transform coefficients. Therefore, our proposed architecture looks like a folded architecture as shown in Fig. 2.

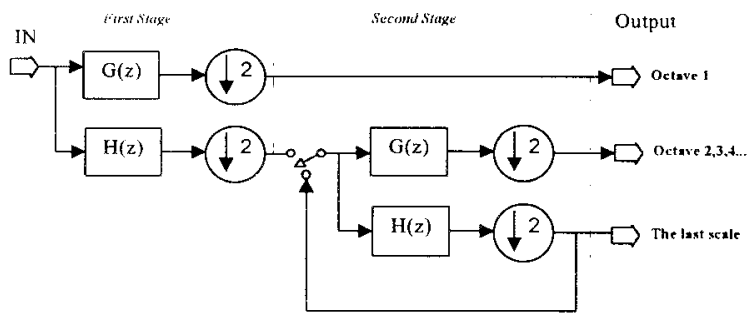

Fig. 2 Overall architecture

The input sequence of the second octave is periodic $(N / 2)$ and generates an output every two clock cycles $(N / 4)$, and the output at the third octave is thus one sample every four clock cycles $(N / 8)$, and so on. The computations of all the octaves after the second octave can be folded into the second-octave filter bank, by cleverly inserting the remaining octave computations into the spacing in the second octave.

Generally, the folded technique is a very good approach to reducing hardware cost for iterative multistage computation, but it may cause a lower I/O rate. Different with the other architectures, where all the computations are folded into a one-octave filter bank, we only folded the octaves after the second. In those methods, they have to interleave the computations of the first octave with those of the other octaves, such that their throughput may not achieve as high enough performance as we did.

\subsection{Decimation Filter Scheme}

As shown in the above section, using our architecture the input data can be computed without being interleaved, but the number of filters used is double. This is beating the benefit of our architecture. To avoid this drawback, instead of using a linear systolic array as the filter bank, we adopt a high-efficient systolic decimation filter architecture similar to the one proposed in [7]. Figure 3 shows such a 4-tap filter used for the computation of input sequence. This filter consists of two submodules, each of which contains one $\mathrm{MAC}$, one register, and two multiplexors (MUX.).

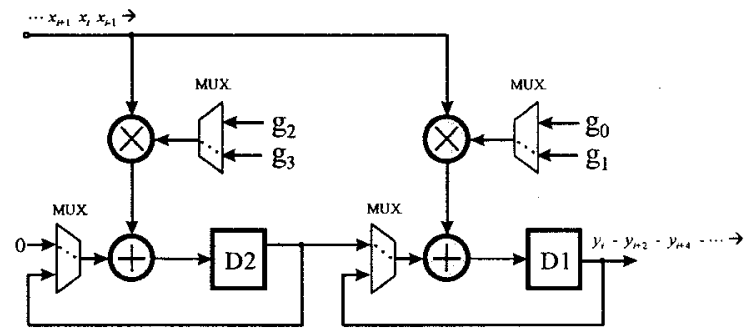

Fig. 3 Systolic decimation filter

Because not every output of the filter banks in Fig. 1 is needed, only one of every two outputs is generated. In Fig. 3. the filter does not generate every output but just the ones that are needed. An operation snapshot of Fig. 3 is shown in Table 1, where the transfer function of the filters is given by:

$$
G(z)=g_{0}+g_{1}^{*} z^{-1}+g_{2}^{*} z^{-2}+g_{3} * z^{-3}
$$

\begin{tabular}{|c|c|c|c|c|c|}
\hline clk & $\mathbf{I} / \mathbf{P}$ & ctrl & D2 & D1 & $\mathrm{O} / \mathrm{P}$ \\
\hline 0 & $\mathrm{X} 0$ & 1 & 0 & 0 & 0 \\
\hline 1 & $\mathrm{X} 1$ & 0 & $g 3 * \times 0$ & g1*X0 & - \\
\hline 2 & $\mathrm{X} 2$ & 1 & $\begin{array}{l}\mathrm{g} 2 * \times 1+ \\
\mathrm{g} 3 * \times 0\end{array}$ & $\mathrm{~g} 0 * \mathrm{XI}+\mathrm{g} 1 * \mathrm{X} 0$ & $\mathrm{~g} 0 * \mathrm{X} 1+\mathrm{g} 1 * \mathrm{X} 0$ \\
\hline 3 & $\bar{x} 3$ & 0 & $\mathrm{~g} 3 * \mathrm{X} 2$ & $\begin{array}{l}\mathrm{g} 1 * \mathrm{X} 2+ \\
\mathrm{g} 2 * \mathrm{X} 1+\mathrm{g} 3 * \mathrm{X} 0\end{array}$ & - \\
\hline 4 & $\overline{X 4}$ & 1 & $\begin{array}{l}\mathrm{g} 2 * \times 3+ \\
\mathrm{g} 3 * \times 2\end{array}$ & $\begin{array}{l}\mathrm{g} 0 * \mathrm{X} 3+\mathrm{g} 1 * \mathrm{X} 2+ \\
\mathrm{g} 2 * \mathrm{X} 1+\mathrm{g} 3 * \mathrm{X} 0\end{array}$ & $\begin{array}{l}\mathrm{g} 0^{*} \mathrm{X} 3+\mathrm{g} 1 * X 2+ \\
\mathrm{g} 2 * X 1+\mathrm{g} 3^{*} X 0\end{array}$ \\
\hline 5 & $\mathrm{X} 5$ & 0 & $\mathrm{~g} 3^{*} \mathrm{X} 4$ & $\begin{array}{l}\mathrm{g} 1 * X 4+ \\
\mathrm{g} 2 * X 3+\mathrm{g} 3 * X 2\end{array}$ & - \\
\hline 6 & $\mathrm{X} 6$ & 1 & $\begin{array}{l}g 2 * X 5+ \\
g 3 * X 4\end{array}$ & $\begin{array}{l}\mathrm{g} 0 * \mathrm{X} 5+\mathrm{g} 1 * \mathrm{X} 4+ \\
\mathrm{g} 2 * \mathrm{X} 3+\mathrm{g} 3 * X 2\end{array}$ & $\begin{array}{l}\mathrm{g} 0 * X 5+\mathrm{g} 1 * X 4+ \\
\mathrm{g} 2 * X 3+\mathrm{g} 3 * X 2\end{array}$ \\
\hline
\end{tabular}

Table 1: Operation snapshot of Fig. 3

In the above filter, only one control signal is used, and the number of multipliers and adders (MACs) is saved by a half compared to the conventional systolic filter, and the register number is saved by a half, too. Therefore, for an $L$-tap filter. the total number of MACs used in our architecture is $2 *(L / 2) * 2=2 L$, which is the same as those architectures proposed in [3]-[6]. The register number is $(L / 2) * 2 * J=L J$ for a $J$-octave DWT, which is much less than the others.

Since the computations in octaves after the second octave are folded into the spacing of the second octave, the secondstage architecture is obviously different from the first stage. Figure 4 shows the second-stage architecture. The operations of the second stage are similar with the first stage. By 
correctly inserting the remaining octave computations into the second-octave spacing, all the computations of the DWT can be accomplished. As seen, the sequence of each octave is generated periodically, so the controller is a simple binary counter. Moreover, each group of octave registers in Fig. 4 is controlled by a selection signal. For scaling up with the filter size, only a simple duplication of submodules is needed to extend the filter for longer-taps.

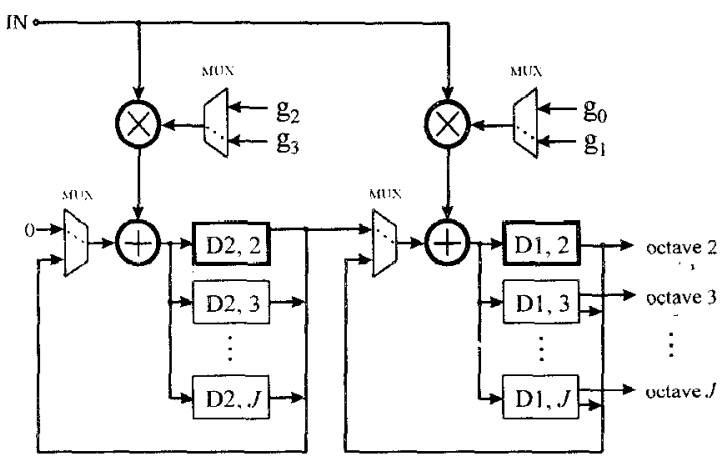

Fig. 4 The second-stage architecture

\section{Chip Design and Implementation}

To verify the performance of the proposed architecture, we have designed and laid out a DWT chip, which number of octaves is three and which filter size is four. In this section, we present the block diagram of the whole chip and the MAC cell. The input, output, and coefficient data are all 2 's complement and 8-bit wide.

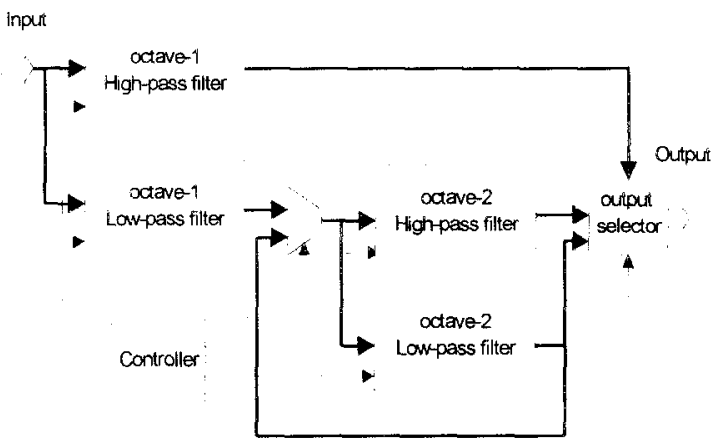

Fig. 5 Block diagram of the chip

Figure 5 shows the block diagram of the whole chip, which consists mainly of two high-pass filters, two low-pass filters, and one controller. The operation of the whole chip has been described in the previous sections. Since the computations of all octave coefficients are interleaved, only one output port is needed, which will save a number of pin count for the chip implementation.

\subsection{Multiplication and Accumulation Cell}

The multiplication and accumulation (MAC) cells are the major components in our design and occupy the most area of the chip. Fig. 6 shows the MAC cell block diagram used in our chip. We combine the multiplier and the adder into one cell. The addition now becomes a partial product of the multiplier. Then the delay time of a multiplier plus an adder, is shortened to that of a multiplier. To further improve the speed, we adopt the modified Booth multiplier [8]-[9] to reduce half of the partial products. For regularity consideration, the partial products are simply added by using carry-save adders. The "final adders" were constructed with a 16-bit carry-select adder having a 4-bit multiplexor based conditional-sum adder as its basic block.

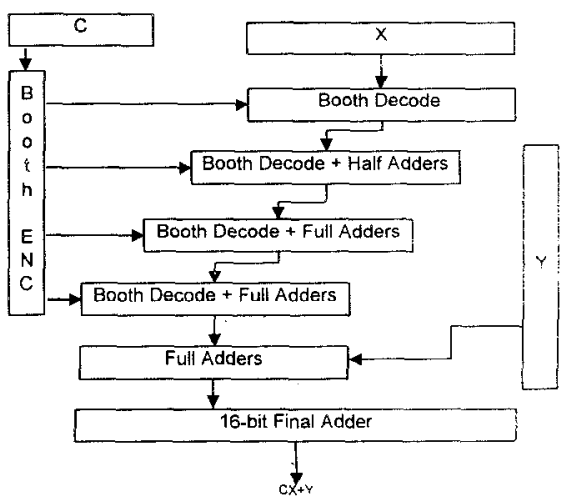

Fig. 6 Block diagram of the MAC cell

After the functional simulation performed using gate-level HDL in a Verilog-XL environment, this chip was synthesized using CADENCE tools and CCL08-V1.0 cell library from the Computer \& Communication Research Laboratories, Hsin-Chu, Taiwan. This chip layout view is shown in Fig. 7. Then, the VLSI chip was fabricated in a $0.8 \mu \mathrm{m}$ Single-Poly-Double-Metal CMOS technology through the Chip Implementation Center, National Science Council, R.O.C. And this chip has been measured with a precise testing machine, IMS Logic Master ATS-200. to verify the intended performance of the proposed 1-D DWT architecture. A snapshot of the testing results was given in Fig. 8. showing that the acquired values are equal to the expected values and that this chip works correctly. The performance data is summarized in Table 2.

Table 2: Performance data

\begin{tabular}{||c|c||}
\hline Transistor Count & 39275 \\
\hline Gate Count & 9231 \\
\hline Core Size & $2974 \times 2868 \mathrm{um}^{2}$ \\
\hline Die Size & $3804 \times 3468 \mathrm{um}^{2}$ \\
\hline Power & $855 \mathrm{~mW}$ \\
\hline Package & $40 \mathrm{pin} \mathrm{DIP}$ \\
\hline Working Frequency & $25 \mathrm{MHz}$ \\
\hline
\end{tabular}

\section{Comparison}

The comparison between our architecture with others is shown in Table 3. We choose only DWT architectures with scalablility to fairly compare each other's performance Following [6], we define some items for the DWT performance. The design cost is the product of the silicon area used by registers and the delay of processing $M$ inpur samples using a $J$ resolution-level discrete wavelet transform. 
and $L$ is the filter length. The improved ratio is generated by dividing the design cost of other architectures with ours. The comparison between our architecture with others is shown in Table 3, where Vishwanath's architectures include two approaches, one systolic and one semi-systolic architectures, respectively [6]. In the table, we see obviously each other's performance according to their design cost and improved ratio.

Table 3: Comparison of various architectures

\begin{tabular}{||c|c|c|c|c||}
\hline Architecture & $\begin{array}{c}\text { Registers } \\
(\mathbf{A})\end{array}$ & $\begin{array}{c}\text { Period/delay } \\
(\mathbf{T})\end{array}$ & $\begin{array}{c}\text { Design cost } \\
(\mathbf{A T})\end{array}$ & $\begin{array}{c}\text { Improved } \\
\text { ratio }\end{array}$ \\
\hline Fridman [5] & $(J+2) L$ & $J M / 2$ & ${ }^{\Omega} L M / 2+L M M$ & $1+J / 2$ \\
\hline Vishwanath [6] & $2 J L, J L$ & $2 M$ & $4 J L M, 2 J L M$ & 4,2 \\
\hline Ours & $J L$ & $M$ & $J L M$ & 1 \\
\hline
\end{tabular}

The comparison of the component numbers of our chip with others is shown in Table 4. All are 4-tap 3-octave 1-D discrete wavelet transform. Comparing with the non-scalable architectures [4], ours uses fewer registers. Comparing with the scalable architectures [5]-[6], ours has higher performance and uses similar hardware components.

Table 4: Component counts of various architectures

\begin{tabular}{||c|c|c|c|c||}
\hline Architecture & MACs & Registers & Input rate & Flexibility \\
\hline Parhi [4] & $8 \mathrm{~m}+6 \mathrm{a}$ & 22 & $1 / 1$ & low \\
\hline Fridman [5] & $8 \mathrm{mac}$ & 18 & $2 / 3$ & high \\
\hline Vishwanath [6] & $8 \mathrm{mac}$ & 24,12 & $1 / 2$ & high \\
\hline Ours & $8 \mathrm{mac}$ & 12 & $1 / 1$ & high \\
\hline
\end{tabular}

\section{Conclusion}

A new high-efficient VLSI architecture for the 1-D DWT decomposition has been described in this paper. Compared to the general folded architecture which folds all the computations into one filter bank, our folded twofilter-bank architecture can achieve high throughput, one sample per clock cycle, for the multi-rate transforms such as discrete wavelet transform. This architecture can also be easily scaled up with the size of the filters and the number of octaves by just using $J L$ registers for a DWT with $J$ octaves and $L$ filter length. The successful implementation of a single chip verifies the intended and anticipated performance of our proposed architecture.

\section{References}

[1] I. Daubechies, Ten Lectures on Wavelets, vol. 61 of CBMS-NSF Regional Conferences Series in Applied Mathematics, SIAM, Philadelphia, PA, 1992.

[2] M. Vetterli, "Wavelet and filter banks: theory and design," IEEE Trans. on Signal Processing, vol. 40, no. 9, pp. 2207-2232, Sep. 1992.

[3] G. Knowles, "VLSI architecture for the discrete wavelet transform," Electronics Letters, vol. 26, no. 15, pp. 1184-1185, July 1990.

[4] K. Parhi and T. Nishitani, "VLSI architecture for discrete wavelet transform, "IEEE Trans. on VLSI Systems, vol. 1, no. 2, pp.191-202, June 1993.
[5] J. Fridman and E. S. Manolakos, "On the synthesis of regular VLSI architecture for the 1-D discrete wavelet transform," Proc. of SPIE Conf. on Mathematical Imaging: Wavelet Applications in Signal and Image Processing 11, San Diego CA, July 1994, pp.296-307.

[6] M. Vishwanath, R. Owens, and M. J. Irwin, "VLSI architecture for the discrete wavelet transform," IEEE Trans. on Circuits and Systems II, analog and digital signal processing, vol. 42, no. 5, pp.305-316, May 1995.

[7] T. Miyazaki, T. Nishitani, M. Ishikawa, M. Edahiro. and K. Mitsuhashi, "Chrominance/Luminance signal separation and syntheses chips developed with a DSP silicon compiler," IEEE Trans. on Circuits and Systems for Video Technology, vol. 2, no. 2, pp. 245-254. June 1992.

[8] P. E. Madrid, B. Millar, and E. E. Swartzlander. Jr. "Modified booth algorithm for high radix fixed Point multiplication," IEEE Trans. on VLSI Systems, vol. 1. no. 2, pp.164-167, June 1993

[9] J. Fadavi-Ardekani, "MxN booth encoded multiplier generator using optimized wallace trees," IEEE Trans. on VLSI Systems, vol. 1, no. 2, pp.120-125. June 1993.

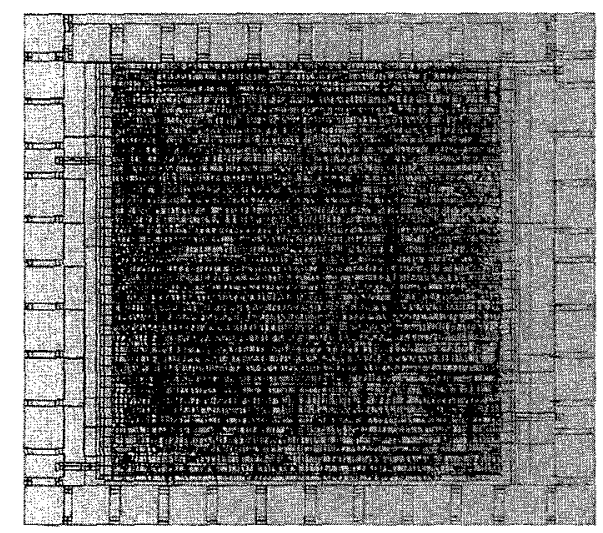

Fig. 7 Chip layout view

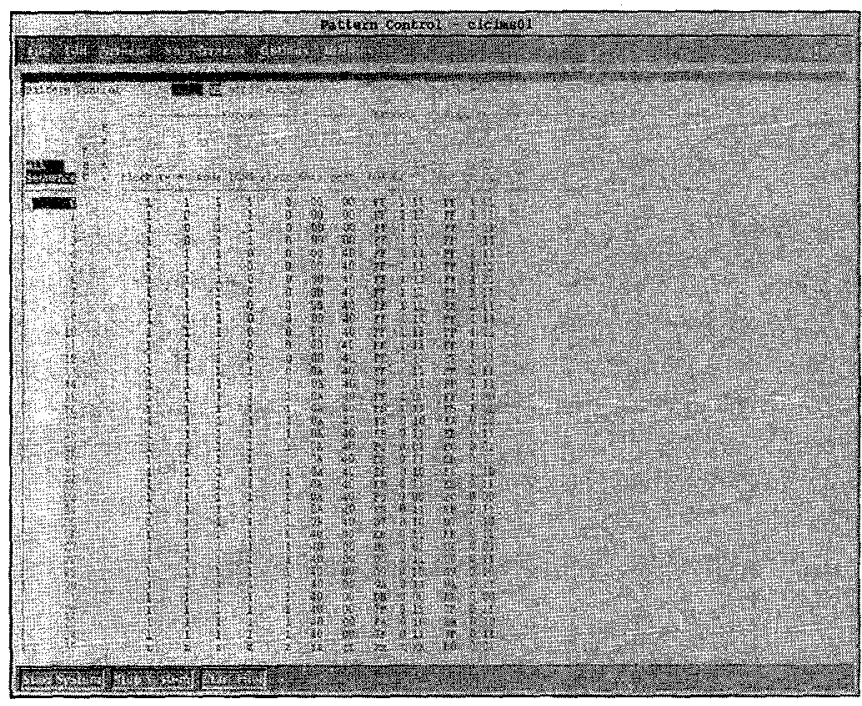

Fig. 8 Snapshot of testing on IMS ATS-200 